\title{
Neumonía varicelosa en el adulto. Estudio de nueve casos
}

\author{
C. PAYTUBÍ, E. NEGREDO, S. FERRER, J. MONMANY, J.L. BARRIO* \\ Servicios de Medicina Interna y Enfermedades Infecciosas. *Hospital de la Santa Creu \\ i Sant Pau. Universitat Autònoma. Barcelona.
}

\author{
PNEUMONIA VARICELLOSA IN THE ADULT. STUDY OF NINE \\ CASES
}

\section{RESUMEN}

Fudamentos: La primoinfección por el virus varicela-zoster en el adulto adquiere una inusitada gravedad debido a complicaciones entre las que la neumonía es la más frecuente. Se estudian nueve pacientes diagnosticados de neumonía varicelosa.

Pacientes y métodos: Se revisan retrospectivamente las peculiaridades clínico-terapéuticas y evolutivas de una serie de pacientes, tanto inmunocompetentes como inmunodeprimidos, diagnosticados en los últimos diez años en el Hospital de Sant Pau de Barcelona. El diagnóstico de varicela se estableció por la aparición del típico rash en el contexto de una enfermedad febril. Se valoran los antecedentes de tabaquismo, embarazo y la presencia de enfermedades de base; analizando especialmente la gasometría arterial y la cifra plaquetar al ingreso.

Resultados: Se estudian 9 pacientes, 4 hombres y 5 mujeres, con una edad media de 38 años. El 78\% eran fumadores de más de 20 cigarrillos/día, uno cumplía criterios de bronquitis crónica, otro tenía una espondilitis anquilopoyética y tres se conocían portadores del virus de la inmunodeficiencia humana. Ninguna de las pacientes estaba embarazada. Los síntomas respiratorios se iniciaron entre el tercer y el quinto día de aparición del exantema, siendo los más frecuentes tos $(89 \%)$, disnea (67\%) y expectoración hemoptoica (22\%). La gasometría arterial evidenció hipoxemia en cuatro (45\%). Asimismo se objetivó trombocitopenia al ingreso en seis $(66 \%)$. La radiografía de tórax mostró un patrón intersticial de predominio bibasal en todos los casos observándose un caso de derrame subpulmonar derecho. Se instauró terapia con aciclovir intravenoso en seis pacientes, foscarnet en uno y tratamiento sintomático en dos. Todos los pacientes siguieron una correcta evolución clínica no precisando ninguno de ellos ser ingresados en la U.C.I.

Conclusiones: Los pacientes adultos con neumonía varicelosa que presentan insuficiencia respiratoria, trombopenia o son portadores de enfermedades de base deben recibir precozmente aciclovir por vía intravenosa. No obstante, si bien la mejoría clínica, biológica y radiológica serían más precoces con dicho tratamiento, la evolución parece igualmente favorable si sólo se instaura, por ejemplo, terapia sintomática con antitérmicos y antihistamínicos.

PALABRAS CLAVE: Neumonía varicelosa. Adultos. Factores de riesgo. Trombocitopenia. Aciclovir.

\section{ABSTRACT}

Background: In the adult, the primary infection by the varicella-zos ter virus acquires an unusual severity due to several complications, the most frequent of them being pneumonia. We study the main characteris tics of nine patients diagnosed of pneumonia varicellosa.

Methods: Clinical, therapeutic and evolutive features of 9 adult patients, both immunocompetents and immunodepressed, diagnosed of pneumonia varicellosa are retrospectively reviewed, in the last ten years, at Hospital de Sant Pau, Barcelona. Diagnosis of varicella was establis hed on the basis of the typical rash in the context of a feverish illness. The antecedents of smoking habit, pregnancy and underlying disease, evaluating especially arterial blood and platelet count at entrance, are assessed.

Results: Nine patients (4 males and 5 women; mean age 38 years) were included in the study. Seventy-eight percent of patients were smo kers of more than 20 cigarettes a day; one met criteria of simple chronic bronchitis, another suffered ankylosing spondylitis and three were known carriers of human immunodeficiency virus. None of the female patients was pregnant. Respiratory symptoms began from the third and fifth day after the skin rash, and the most common symptoms were cough (89\%), dyspnea (67\%) and hemoptysis (22\%). Arterial blood gas deter mination showed hypoxemia in four patients $(45 \%)$. Chest $X$-ray revea led an interstitial pattern predominantly at both bases, with a case of right pleural effusion. Intravenous acyclovir was started in 6 patients, foscarnet in one and symptomatic therapy in two patients. All patients had a favourable clinical course, none of them requiring entrance to the Intensive Care Unit.

Conclusions: Adult patients with varicella pneumonia that suffer res piratory insufficiency, thrombocytopenia or are carriers of base illnesses must be early treated with intravenous acyclovir. However, despite clini cal, biological and radiological recovery is earlier with such treatment, the evolution seems equally favourable if it is only conducted, for instan ce, symptomatic therapy with antitermic and antihystaminic compounds.

KEY WORDS: Varicella-pneumonia. Adult. Risk factors. Thrombocyto penia. Acyclovir.

Paytubí C, Negredo E, Ferrer S, Monmany J, Barrio JL. Neumonía varicelosa en el adulto. Estudio de nueve casos. An Med Interna (Madrid) 2001; 18: 312-316.

Trabajo aceptado: 8 de Noviembre de 1999

Correspondencia: Dr. Carlos Paytubí Garí. Servicio de Medicina Interna. Centro Médico Teknon. Barcelona 


\section{INTRODUCCIÓN}

La varicela es la forma de presentación habitual de la primoinfección por el virus Varicela-Zoster. Se trata de una infección generalmente benigna, propia de niños menores de 10 años (máxima incidencia entre los 2 y los 8 años). Sin embargo, en la edad adulta (5\% de los casos) puede adquirir una notable gravedad debido a complicaciones neurológicas, hematológicas y respiratorias. Entre sus complicaciones, la neumonía es la más común y la que presenta mayor gravedad (1-3), recogiéndose una mortalidad, variable según las series, en el adulto sano, del 10 al 30\%, incrementándose este porcentaje en el caso de los pacientes inmunocomprometidos $(4,5)$.

En la mayor parte de los casos la neumonía varicelar incide por encima de los 20 años, alcanzando la máxima frecuencia entre la segunda y la quinta década de la vida (6).

En casi todos los pacientes es posible constatar el antecedente epidemiológico de contacto con niños afectos de varicela, estableciéndose la sospecha diagnóstica por la aparición del típico rash en el contexto de un cuadro febril. Las formas clínicas incluyen desde la forma asintomática hasta cuadros graves con severa insuficiencia respiratoria, siendo precisamente en estos últimos casos así como cuando se asocian factores de mal pronóstico (enfermedad pulmonar obstructiva crónica, diabetes mellitus, inmunodepresión, alcoholismo) cuando debe valorarse la terapia con aciclovir, cuya eficacia, aplicada de modo precoz, ha quedado plenamente demostrada $(7,8)$.

Presentamos una serie retrospectiva de nueve casos de neumonía varicelosa en adultos, valorando las características clínicas, radiológicas, epidemiológicas y bioquímicas, destacando la utilidad del aciclovir por vía intravenosa en aquellas que cursan con insuficiencia respiratoria.

\section{CASOS APORTADOS}

Se revisaron retrospectivamente las historias clínicas de nueve pacientes adultos, tanto inmunocompetentes como inmunodeprimidos, que, procedentes de urgencias, fueron ingresados en el Servicio de Medicina Interna de un Hospital Universitario en los últimos diez años afectos de neumonía varicelar. No hubieron criterios predefinidos de ingreso ni edad de corte que excluyese pacientes para el estudio. Se estableció el diagnóstico por criterios clínico-radiológicos en el curso de una varicela, definida por la aparición de un exantema vesiculoso generalizado en el contexto de un síndrome febril. Se recogieron antecedentes epidemiológicos de contacto con pacientes afectos de varicela, hábitos tabáquico y enólico, enfermedades de base y situación de competencia inmunológica. Se valoraron asimismo datos de la historia clínica, exploración física, bioquímica sanguínea (con especial relevancia en el recuento plaquetar al ingreso), radiología de tórax, gasometría arterial, tratamientos administrados y evolución posterior.

Se estudiaron un total de nueve pacientes con neumonía varicelosa, cuatro varones y cinco mujeres, con edades comprendidas entre los 29 y los 48 años, con una media de edad de 38 . Uno cumplía criterios de bronquitis crónica simple, otro tenía una espondiloartritis anquilopoyética HLA B27 corticodependiente y tres estaban afectos del síndrome de inmunodeficiencia adquirida en fase avanzada $\left(\mathrm{CD} 4<50 / \mathrm{mm}^{3}\right)$, estando uno de ellos afecto de una hepatopatía crónica por virus C. De los nueve pacientes, siete (78\%) presentaban un consumo de tabaco igual o superior a un paquete diario, y dos de ellos además, una ingesta enólica superior a 60 gramos/día. Ninguna de las cinco pacientes estaba embarazada. Se pudo recoger el antecedente epidemiológico de contacto con niños

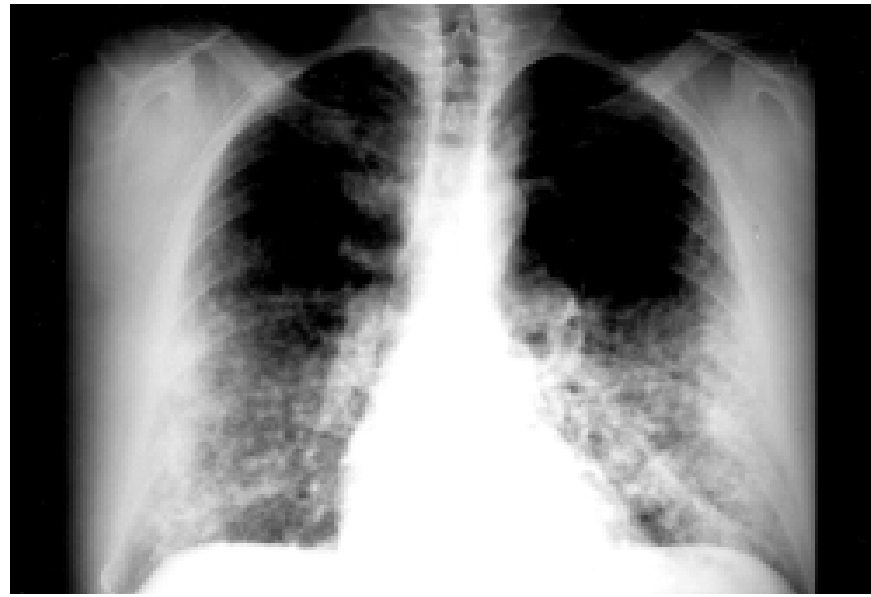

Fig. 1. Patrón retículonodulillar de predominio bibasal característico de neumonía varicelosa (paciente número 1 en el momento del diagnóstico).

afectos de varicela en cuatro de los casos (45\%); clínicamente en todos ellos se observó fiebre superior a $38^{\circ} \mathrm{C}$ (alcanzándose en dos de los casos $40^{\circ} \mathrm{C}$ ) y la aparición del típico rash pruriginoso a los pocos días del inicio del cuadro febril. La sintomatología respiratoria se inició entre el tercer y el quinto día de la aparición del exantema, y consistió en tos no productiva en ocho individuos (89\%), disnea en seis $(67 \%)$, dolor torácico de características pleuríticas en uno $(11 \%)$ y expectoración hemoptoica en dos $(22 \%)$. Los hallazgos físicos fueron mínimos y se correlacionaron pobremente con el grado de severidad de la neumonía, lo que define la típica disociación clínico-radiológica de las neumonías atípicas. En todos los casos la radiología de tórax evidenció un patrón retículonodulillar bilateral con tendencia a unirse cerca del hilio y en las bases pulmonares, detectándose en uno de los casos un derrame subpulmonar derecho. La gasometría arterial mostró una hipoxemia en cuatro casos $(45 \%)$. El recuento plaquetar al ingreso fue inferior a $150 \mathrm{x}$ $10^{9} / 1$ en seis de los casos (66\%) siendo en un caso de $43 \times 10^{9} / 1$, no evidenciándose en ninguno de ellos manifestaciones discrásicas como epistaxis o petequias. Ninguno de los pacientes, incluso aquellos con importante insuficiencia respiratoria $\left(\mathrm{pO}_{2}\right.$ de hasta 44 $\mathrm{mmHg}$ ), requirió ingreso en la UCI. Se instauró tratamiento con aciclovir por vía intravenosa a dosis de $5 \mathrm{mg} / \mathrm{Kg} / 8 \mathrm{~h}$ en inmuno-

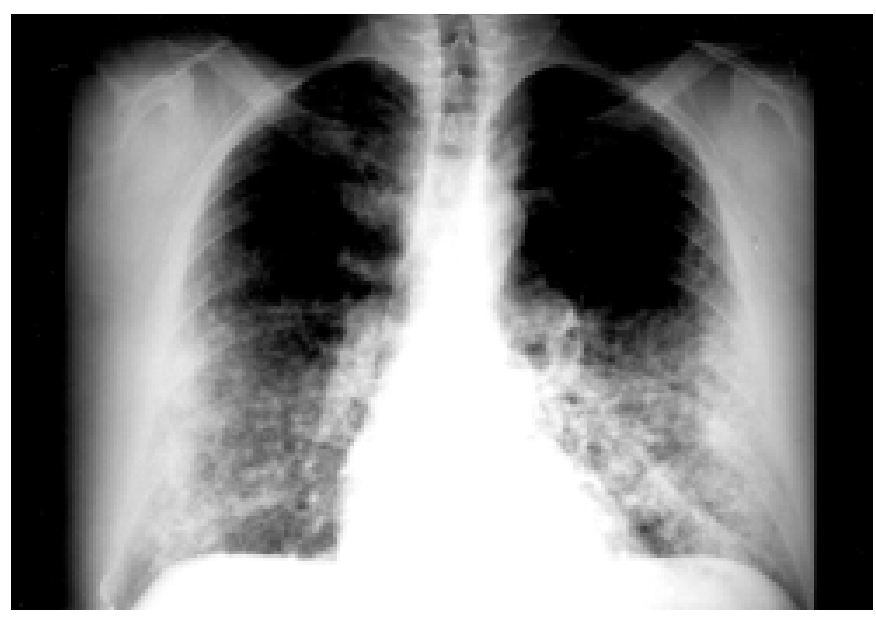

Fig. 2. TAC torácica del mismo paciente mostrando afectación difusa y bilaterla caracterizada por la presencia de imágenes nodulillares de contornos mal definidos. 


\begin{tabular}{|c|c|c|c|c|c|c|c|c|c|}
\hline \multicolumn{10}{|c|}{ TABLA I } \\
\hline \multicolumn{2}{|c|}{ Número Sexo/Edad } & Contacto & Hábitos & Enfermedad & Síntomas & $\mathrm{pO}_{2}$ & Plaquetas & U.C.I. & Tratamiento \\
\hline 1 & $\mathrm{H} / 42 \mathrm{a}$ & No & Tabaco & Epoc & $\begin{array}{c}\text { Tos } \\
\text { Disnea } \\
\text { Dolor pleurítico }\end{array}$ & $\begin{array}{c}44 \\
\mathrm{mmHg}\end{array}$ & 65.000 & No & $\begin{array}{l}\text { Aciclovir } \\
\text { ev }\end{array}$ \\
\hline 2 & $M / 35 \mathrm{a}$ & Sí & Tabaco & $\begin{array}{c}\text { Espondilitis } \\
\text { Anquilopoyética }\end{array}$ & $\begin{array}{c}\text { Tos } \\
\text { Disnea }\end{array}$ & 66 & 149.000 & No & $\begin{array}{l}\text { Aciclovir } \\
\text { ev }\end{array}$ \\
\hline 3 & $M / 35 \mathrm{a}$ & Sí & Tabaco & & $\begin{array}{c}\text { Tos } \\
\text { Disnea } \\
\text { Expectoración } \\
\text { hemoptoica }\end{array}$ & 56 & 80.000 & No & $\begin{array}{l}\text { Aciclovir } \\
\text { ev + vo }\end{array}$ \\
\hline 4 & $\mathrm{H} / 48 \mathrm{a}$ & Sí & $\begin{array}{l}\text { Tabaco } \\
\text { Alcohol }\end{array}$ & & & 68 & 270.000 & No & Sintomático \\
\hline 5 & $M / 29 a$ & Sí & Tabaco & & $\begin{array}{c}\text { Tos } \\
\text { Disnea } \\
\text { Expectoración } \\
\text { hemoptoica }\end{array}$ & 57 & 180.000 & No & Sintomático \\
\hline 6 & $M / 32 a$ & No & Tabaco & & Tos & 85 & 43.000 & No & $\begin{array}{c}\text { Aciclovir } \\
\text { ev }\end{array}$ \\
\hline 7 & $\mathrm{M} / 30 \mathrm{a}$ & No & Tabaco & $\mathrm{VIH}+$ & Tos & 73 & 150.000 & No & $\begin{array}{l}\text { Foscarnet } \\
\text { ev }\end{array}$ \\
\hline 8 & $\mathrm{H} / 28 \mathrm{a}$ & No & Tabaco & VIH + & $\begin{array}{c}\text { Tos } \\
\text { Disnea }\end{array}$ & 84 & 115.000 & No & $\begin{array}{l}\text { Aciclovir } \\
\text { ev }\end{array}$ \\
\hline 9 & $\mathrm{H} / 35 \mathrm{a}$ & No & $\begin{array}{l}\text { Tabaco } \\
\text { Alcohol }\end{array}$ & $\begin{array}{l}\mathrm{VIH}+ \\
\mathrm{VHC}+\end{array}$ & $\begin{array}{c}\text { Tos } \\
\text { Disnea }\end{array}$ & 49 & 169.000 & No & $\begin{array}{l}\text { Aciclovir } \\
\text { ev }\end{array}$ \\
\hline
\end{tabular}

competentes y de $10 \mathrm{mg} / \mathrm{Kg} / 8 \mathrm{~h}$ en inmunodeprimidos, comprobándose una respuesta satisfactoria en todos ellos, lográndose la apirexia entre el segundo y el cuarto día y objetivándose una resolución progresiva del cuadro respiratorio. Sólo en un caso -paciente con síndrome de inmunodeficiencia adquirida con severa deplección linfocitaria $\left(C D 4=4 / \mathrm{mm}^{3}\right)$ - el equipo médico responsable aplicó terapia antivírica con foscarnet intravenoso. La duración del tratamiento osciló entre siete y doce días. En los restantes pacientes, en los que la neumonía fue un hallazgo radiológico y que sólo recibieron tratamiento sintomático, la evolución fue igualmente favorable teniendo en cuenta que no presentaron repercusión gasométrica ni trombopenia. En la tabla I se recogen las principales características de los nueve pacientes.

\section{DISCUSIÓN}

La primoinfección por el virus varicela-zoster se presenta en la mayoría de los casos en forma de varicela. Se trata de una infección exantemática generalmente de curso benigno y autolimitada, salvo en recién nacidos e inmunodeprimidos, propia de la edad pediátrica. No obstante, en la edad adulta el proceso puede adquirir una notable gravedad debido fundamentalmente a complicaciones (9) neurológicas (encefalomielitis aguda postinfecciosa, ataxia cerebelosa aguda, meningitis aséptica, neuritis óptica, mielitis transversa), hematológicas (hemorragias, púrpura trombocitopénica aguda) y respiratorias (neumonía varicelosa). Otras complicaciones descritas son las cutáneas, oculares, cardiacas, articulares, renales, urológicas, y excepcionalmente otorrinolaringológicas (supraglotitis aguda) u óseas (osteomielitis).
En relación a las complicaciones hematológicas, si bien los fenómenos hemorrágicos asociados a la varicela son muy infrecuentes, la trombocitopenia suele ser la norma en un alto porcentaje de los casos (en nuestra serie fue del 66\%). Sin embargo, existe mucha controversia acerca del mecanismo etiopatogénico implicado desde que ya en la década de los 60 se argumentaba con una coagulación intravascular diseminada responsable de la destrucción plaquetar (10). Recientes estudios publicados $(11,12)$ describen mecanismos autoinmunes como la detección de autoanticuerpos dirigidos contra la glicoproteína de superficie $\mathrm{V}$ de las plaquetas.

La neumonía varicelosa constituye la complicación más grave y frecuente en la edad adulta, con una mortalidad variable según la series (13), pudiendo ser en el adulto sano de hasta el $30 \%$, aumentando exponencialmente este porcentaje en los pacientes inmunodeprimidos.

La neumonía varicelosa fue descrita por primera vez en 1942 por J.J. Waring (14), pudiéndose presentar con un amplio espectro clínico, desde formas banales y asintomáticas diagnosticadas únicamente a partir de los hallazgos radiológicos, hasta formas con severa insuficiencia respiratoria que pueden llegar a requerir intubación orotraqueal, ventilación mecánica e ingreso en una UCI con una mortalidad muchas veces superior al $30 \%$.

Diversos factores de riesgo han sido descritos en la neumonía varicelosa del adulto, reflejando algunos de ellos (embarazo, EPOC previa) una mayor gravedad pronóstica:

1. Edad: Mayor severidad y afectación radiológica en pacientes más añosos (15).

2. Hábito tabáquico: Altera los macrófagos alveolares 
haciéndolos más susceptibles a la infección por herpes virus (16), con el consiguiente desarrollo de neumonitis, calculándose que el riesgo relativo de aparición de ésta en los fumadores es quince veces mayor que en los no fumadores, al igual que el posterior desarrollo de las calcificaciones pulmonares (17). Asimismo, estudios de función respiratoria durante la neumonía varicelosa y después de la misma, muestran, en los fumadores, una reducción del coeficiente de transferencia del $\mathrm{CO}_{2}$ y del factor de difusión que puede persistir años después (18). En nuestra serie se confirma esta asociación de riesgo en el $100 \%$ de los casos.

3. Embarazo: Sin que hasta la fecha se conozcan los mecanismos etiopatogénicos implicados, la aparición de lesiones cutáneas típicas de la infección por el virus varicela-zoster es criterio de ingreso hospitalario e inicio de tratamiento intravenoso con aciclovir, no sólo por el riesgo de fetopatía (hasta del $10 \%$ ), sino por la posibilidad de insuficiencia respiratoria grave (19-22).

4. Enfermedad Pulmonar Obstructiva Crónica: Mayor severidad de complicaciones pulmonares que los individuos sanos (14). En nuestra serie el paciente que cumplía los criterios presentaba el mayor grado de hipoxemia al ingreso $\left(\mathrm{pO}_{2}=44 \mathrm{mmHg}\right)$.

5. Enfermedades de base que cursen con inmunodepre sión, sobre todo neoplasias hematológicas y tratamientos quimioterápicos (23).

6. Rash cutáneo: Correlación bien definida entre la severidad de la neumonía y el grado de afectación cutánea y/o carácter hemorrágico de las lesiones (24).

La infección por el virus varicela-zoster en las personas infectadas por el VIH es frecuente (25-27); sin embargo, a pesar de la frecuencia con la que se presenta la forma cutánea y de la importante disfunción del sistema inmunitario (CD4 $<25 / \mathrm{mm}^{3}$ en los tres casos de nuestra serie), la neumonía varicelosa se ha descrito sólo en casos aislados en estos pacientes $(25,28)$.

La neumonía varicelosa se suele presentar entre el segundo y el sexto día tras la aparición del exantema, en forma de tos seca o escasamente productiva, fiebre que puede sobrepasar los $39^{\circ} \mathrm{C}$ y disnea de hasta mínimos esfuerzos, siendo menos frecuente la presencia de dolor torácico (generalmente de características pleuríticas) o artromialgias, y ocasional la hemoptisis $(3,5,14,29)$. Puede haber dolor dorsal o abdominal, incluso como el primer signo clínico, simulando, a veces, cuadros de abdomen agudo (30). Todos nuestros pacientes presentaron sintomatología respiratoria, fundamentalmente tos, fiebre y disnea. Los hallazgos semiológicos son generalmente escasos y se correlacionan mal con el grado de afectación radiológica (disociación clínico-radiológica) (6). La gasometría arterial puede mostrar, desde valores dentro del rango de la normalidad hasta severa hipoxemia en las formas no necesariamente más graves. Los hallazgos radiológicos más frecuentes son los infiltrados bilaterales, difusos, reticulonodulillares, que tienden a coalescer en los hilios y bases respetando las zonas periféricas del parénquima pulmonar y que pueden confluir dando grandes condensaciones, incluso con broncograma aéreo. Esta imagen radiológica no se correlaciona bien con la severidad de la enfermedad, pudiéndose acompañar ocasionalmente de derrame pleural uni o bilateral (14-16,24,31). En general, la mejoría del cuadro clínico se inicia con la resolución del exantema, pero la mejoría radiológica puede demorarse dos o más semanas, o bien, persistir lesiones fibróticas, o incluso, llegar a la calcificación miliar de los pequeños nódulos (32).

Con respecto al tratamiento, algunos estudios sugieren la efectividad del aciclovir por vía intravenosa, siempre y cuando se inicie de un modo precoz, observándose una respuesta clínica en 24-48 horas (33), aunque no está claro que altere, al menos en todos los casos, el curso de la neumonía, dado que hay pacientes que sin tratamiento mejoran rápidamente $(3,16,34)$. El virus varicela-zoster es menos sensible que el virus herpes simple al aciclovir, pero se inhibe bien a las dosis habitualmente utilizadas, que oscilan entre 5 y $10 \mathrm{mg} / \mathrm{Kg} / 8 \mathrm{~h}$ durante siete a diez días $(33,35)$. Los pacientes con sintomatología respiratoria y/o hipoxemia fueron tratados con aciclovir intravenoso con dosis de 5 a $10 \mathrm{mg} / \mathrm{kg} / 8 \mathrm{~h}$, dependiendo del estado inmunitario, con una correcta evolución. Ninguno de nuestros pacientes, incluso uno de ellos que recibió tratamiento sintomático, pese a objetivarse importante insuficiencia respiratoria con hipoxemia de $57 \mathrm{mmHg}$, requirieron ingreso en UCI. En nuestra serie, por tanto, si bien los pacientes que recibieron aciclovir por vía intravenosa evidenciaron una respuesta clínica más precoz, no pueden extraerse diferencias evolutivas estadísticamente significativas entre los que recibieron tratamiento antivírico (78\%) y los que recibieron únicamente tratamiento sintomático $(22 \%)$ con antitérmicos y antihistamínicos. Otra alternativa a considerar descrita recientemente (36) es la de realizar el tratamiento de la neumonía varicelosa con buen estado general y nula o muy escasa repercusión ventilatoria con aciclovir por vía oral en régimen ambulatorio bajo estrecho control clínico y aleccionando al paciente sobre los síntomas que deberían hacerle acudir al hospital sin demora. En esta línea, si bien la evolución observada ha sido correcta, son necesarios más estudios controlados que establezcan la indicación de este régimen terapéutico que evita la hospitalización. Igualmente se ha señalado la dificultad de alcanzar niveles séricos adecuados con el empleo de la vía oral (37).

En conclusión y a la vista de los resultados obtenidos, del análisis realizado, de la escasa toxicidad observada con el aciclovir (nefropatía obstructiva por cristalización del fármaco en los túbulos renales, trastornos digestivos, flebitis y síntoma neurológicos como desorientación y temblores, entre otros) y de la revisión de otras series de pacientes con neumonía varicelosa (38-42), parece que, como asimismo recomienda el CDC americano, en los pacientes tanto inmunocompetentes como inmunodeprimidos que desarrollan sintomatología respiratoria e hipoxemia en el curso de la varicela, debe instaurarse de un modo precoz tratamiento con aciclovir por vía intravenosa pues si bien no sería imprescindible en todos los casos, sí parece evidente que la mejoría clínica y de la cifra de plaquetas es más precoz, lográndose en prácticamente todos los casos evitar la aparición de signos de compromiso respiratorio con el consiguiente traslado a una Unidad de Vigilancia Intensiva. 


\section{Bibliografía}

1. Weber D, Pellechia J. Varicella pneumonia. JAMA 1965; 192: 572-573.

2. Bogles JS, Ehrenkranz NJ, Marks A. Abnormalities of respiratory function in varicella pneumonia. Ann Intern Med 1964; 60: 183-195.

3. Schlossberg D, Litman M. Varicella pneumonia. Arch Intern Med 1988; 148: 1630-1632.

4. Guess HA. Population-based studies of varicella complications. Pediatrics 1986; 78: 723-727.

5. Wenstein L, Meade R. Respiratory manifestations of chicken pox. Arch Intern Med 1956; 98: 91-99.

6. Molina M, Ortega G, García B, Pérez A, Sáez JA, De Paco M. Neumonía por varicela en adultos previamente sanos. Rev Esp Microbiol Clin 1989; 4: 712-714.

7. Davidson RN, Lynn W, Savage P, Wnasbrough-Jones MH. Chickenpox pneumonia: experience with antiviral treatment. Thorax 1988; 43: 627-630.

8. García Quintana A, Alegre J, Falcó V, Fernández de Sevilla T, Martínez Vázquez JM. Neumonía varicelosa en el adulto. Estudio de trece casos. Rev Clin Esp 1992; 191: 314-316.

9. Straus S, Ostrove J, Inchauspé G, Felser J, Freifeld A, Croen K, Sawyer M. Varicella Zoster Virus infections: Biology, Natural History, Treatment and Prevention. Ann Intern Med 1988; 88: 221-237.

10. McKay DG, Margaretten W. Disseminated intravascular coagulation in virus diseases. Arch Intern Med 1967; 120: 129-152.

11. Mayer J, Beardsley D. Varicella-Associated Thrombocytopenia: Autoantibodies against Platelet Surface Glycoprotein V. Pediatric Res 1996: 40: 615-619.

12. Fraser Wright J, Blanchette V, Wang H, Arya N, Petric M, Semple J, Chia W-K et al. Characterization of platelet-reactive antibodies in children with varicella-associated acute immune thrombocytopenic purpura. Br J Haematol 1996; 95: 145-152.

13. Pugh RN, Omar RI, Hossain MM. Varicella infection and pneumonia among adults. Int $\mathrm{J}$ Infect Dis 1998; 2: 205-10.

14. Waring JJ, Neubuerger K, Greever EF. Severe form of chickenpox in adults. Arch Intern Med 1942; 69: 384-408.

15. Chitkara R, Gordon R, Khen F. Acyclovir in the treatment of primary varicella pneumonia in non immunocompromised adults. NY State J Med 1987; 237-238.

16. Rose RM, Wasserman AS, Weiser W, Reinhold HG. Deficient responses of pulmonary macrophages from healthy smokers to antiviral lymphokines in vitro. J Infect Dis 1986; 154: 611.

17. Ellis M, Neal K, Weeb A. Is smoking a risk factor for pneumonia in adults with chickenpox? Br Med J 1987; 294: 1002.

18. Ehrenkranz NJ, Mark A, Bogles JS. Abnormalities in respiratory function in varicella pneumonia. Ann Intern Med 1964; 60: 183-195.

19. Boyd K, Walker E. Use of acyclovir to treatment chickenpox in pregnancy. Br Med J 1988; 296: 393-394.

20. White R. Chickenpox in pregnancy. Br Med J 1988; 296: 864.

21. Chodos WS. Varicella in pregnancy: Report of a case and review of literature. J Am Osteopath Assoc 1982; 81: 644-666.

22. Rodrigues J, Niederman MS. Pneumonia Complicating pregnancy. Clin Chest Med 1992; 13: 679-691.

23. Ballow M, Hirschhorn. Varicella pneumonia in a bone marrow-trasplanted, immune-reconstituted adenosine deaminase deficient patient with severe combined immunodeficiency disease. J Clin Immunol 1985; 5: 180-186.
24. Gogos CA, Bassaris HP, Vagenakis AG. Varicella pneumonia in adults. A review of pulmonary manifestations, risk factors and treatment. Respiration 1992; 59: 339-343.

25. Sans M, Gatell JM, Rafael M, Mallolas J, Soriano E. Neumonía varicelosa en adultos infectados por el VIH-1. Presentación de dos casos. Enferm Infecc Microbiol Clin 1994; 12: 26-30.

26. Quinnan GV, Masur H, Rook AH. Herpesvirus infections in the acquired immune deficiency syndrome. JAMA 1984; 252: 72-77.

27. Leibovitz E, Cooper D, Giurgiutiu D, Coman G, Straus I, Orlow S, Lawrence R. Varicella-Zoster Virus Infection in Romaninan Children Infected With the Human Immunodeficiency Virus. Pediatrics 1993; 92: 838- 842.

28. Muñoz A. Sobre la neumonía varicelosa. Enferm Infecc Microbiol Clin 1994; 12: 58.

29. Feldman S. Varicella-zoster virus pneumonitis. Chest 1994; 106: 22S$27 \mathrm{~S}$.

30. Triebwasser JH, Harris RE, Bryant RE, Rhoades ER. Varicella pneumonia in adults. A report of seven cases and a review of the literature. Medicine 1967; 46: 409-423.

31. Sargent N, Carson MJ, Reilly ED. Roentgenographic manifestations of varicella pneumonia with postmortes correlation. Am J Roentgenol Rad Ther Nucl Med 1966; 98: 305-317.

32. Meyer B, Stalder H, Wegmann W. Persistent pulmonary granulomas after recovery from varicella. Chest 1986; 89 (3): 457-459.

33. Van der Meer JWM, Thompson J, Tan WD, Versteeg J. Treatment of chickenpox pneumonia with acyclovir. Lancet 1980; ii: 473-474.

34. Olivé A, Estrems M, Vallés J, Albert J, Betriu N. Neumonía varicelosa y aciclovir. Med Intensiva 1990; 14: 177-178.

35. Haake DA, Zakowski PC, Haake DL, Bryson YJ. Early treatment with acyclovir for varicella pneumonia in otherwise healthy adults: retrospective controlled study and review. Rev Infect Dis 1990; 12: 788798.

36. Gil Sanz E, Arribas JM, López A, González-Baylin ML. Neumonía por varicela zoster. Tratamiento por vía oral con aciclovir. Enferm Infecc Microbiol Clin 1995; 13: 193-194.

37. MacKendrick et al. BMJ 1986; 293:1529-32.

38. Alemán C, García Quintana AM, Alegre J, Recio J, Falcó V, Fernández de Sevilla T. Neumonía varicelosa en el adulto. Revisión de 25 casos. Rev Clin Española 1997; 197: 690-692.

39. Fernández Vázquez E, Soudan A, Espejo A, González Vargas F, Cabrera Torres L. Neumonía varicelosa en un adulto sano. Revisión de factores de riesgo y tratamiento. An Med Interna (Madrid) 1994; 4: 187-188.

40. Escalante M, Franco-Vicario R, Cisterna R, Solano D, Miguel De La Villa F, Imaz M. Neumonía por varicela en el adulto. An Med Interna (Madrid) 1994; 5: 244-246.

41. Pérez-Llorens JC, Crusells MJ, Araiz JJ, Letona S, Millastre A, Cuesta J. Síndrome de distress respiratorio del adulto secundario a neumonía varicelosa en varón inmunocompetente. An Med Interna (Madrid) 1994; 4: 189-191.

42. Golpe Gómez R, Garcia Arangüena L, Garcia Pérez MM, De la Roza Fernández CO, Fernández Rozas SM, Cifrián Martínez JM. Neumonía varicelosa en el adulto previamente sano. An Med Interna (Madrid) 1999; 16: 83-86. 